Committee has been appointed to deal with the standards of vision for scholars and teachers in Council Schools.

During the course of the year several questions have been referred to the Council for its opinion and advice. Amongst these may be mentioned that of lectures to candidates for the examinations of the British Optical Association and Spectacle Makers Co., by the Universities of Glasgow and Liverpool; also proposals concerning sight-testing by opticians under the National Insurance Act.

Another important question which the Council has had under consideration is the establishment of an International Year Book of Ophthalmology in English, proposed by Ophthalmologists in America.

The expenses of the Council have been defrayed as in previous years by its members.

The Council has to thank the Council of the Royal Society of Medicine for the use of a room for its meetings.

\title{
ABSTRACTS
}

\section{I.-LATE RESULTS OF SCLERECTO-IRIDECTOMY}

Lagrange and Baron.-Remote results of sclerecto-iridectomy in the treatment of chronic glaucoma. (Résultats éloignés de la sclérecto-iridectomie dans le traitement du glaucome chronique.) Arch. d'Ophtal., January, 1923.

The eminence of the senior author and the precision with which he and his assistant have collated the results of 10 years' work give exceptional value to this article. It covers 40 pages, presents five principal headings, and abounds in figures; the following is merely a summary.

1. Precision in terminology. Lagrange's well-known operation comprises excision of tissue from sclera and from iris, and the author's name for it is correct. The term " irido-sclerectomie," substituted by certain writers, is faulty for it does not indicate the treatment of the iris; true, it is shorter by one syllable and a little easier to say, but precision should not be sacrificed to brevity or euphony.

2. What are to be taken as remote results? In general surgery cicatrices change long after the lips of the wound are joined, and the same is true of incisions in the eye. In chronic glaucoma success depends on lasting fistulization of the eye, and at least one year should pass before the result is reported. All cases included in these statistics were re-examined at least one year after the 
operation, and many of them much later, for they represent a period of 10 years' work.

At the London Congress of 1913 Lagrange reported his results for the previous decade, under the same restriction. They are not exactly comparable with those of the later period, for among the earlier cases were a considerable number in which simple sclerectomy (i.e., without iridectomy) was employed, but a brief restatement of them may be given here:-Of 104 patients 15 showed lowered visual acuity when re-examined, but in 8 of these it was due to development of cataract long after the operation, and in 4 of these latter the cataract was extracted later with success; of the 7 cases of actual non-success, one was an acute glaucoma, 2 showed further atrophy of the nerve, 1 presented " inhibition rapide," 1 retinal haemorrhage, 1 recurrent haemorrhage into anterior chamber, 1 failed because the sclerectomy was too small.

These 104 cases were those which were examined after at least one year; the total number operated on was 240 , and among the whole of these not a single case of infection, loss of vitreous, expulsive haemorrhage, or traumatic cataract was seen.

3. Cases not previously published. During the 10 years 1913 to 1923 the great war threw new duties on the Chief and his Staff, and while the number of cases treated was larger, the proportion which could be followed closely enough for inclusion in these statistics was smaller than before. In all the included cases the acuity of vision and the field were carefully examined before the operation; in some the tension was tested only with the finger, but in most of them the tonometer was used. They were 49 in number and comprised 68 operations. In a few the iridectomy was a peripheral button-hole, but in most it was complete; 4 of the cases were of irritative type, presenting slight congestion with frequent well-marked rises of tension; 28 were chronic with persistent excess; 17 were chronic with intermittent excess. No case of acute glaucoma was treated by sclerecto-iridectomy.

Here follows a detailed statement of the cases covering 16 pages.

4. Results. Acuity of vision showed no further loss in 25 eyes, and to this group may be added 3 more in which it fell only a little in accordance with advancing age; in 18 it rose; in 17 it fell. In 5 cases vision was already lost before the operation, which was performed merely to reduce tension. In 6 cases the loss of acuity was due to gradual development of senile cataract not attributable in any way to the operation, for the tension remained normal and the projection good. Reckoning these with the good results, the successes amounted to 82.5 per cent. The non-successes appeared to depend on continued atrophy of the nerve in spite of normalized tension.

The results as regards tension relate only to cases measured 
with the tonometer, 40 in number. It was normalized, i.e., it lay between 15 and 27 (Schiötz), in 32 cases, but in this number are included 2 in which it stood at 30 (Schiötz), for the functional results in these were excellent. Slight hyper-tension was found in 5 cases and appeared to be due to insufficient removal of scleral tissue. Slight hypo-tension was found in 4 cases. Some authors regard this as no disadvantage; Lagrange considers a tension below 15 (Schiötz) as likely to prejudice the eye. The successes as regards tension were therefore 80 per cent., or 87.5 if hypotensions are included.

No case was seen of infection, sympathetic ophthalmia, traumatic cataract, or wound of the ciliary body.

5. Comparison with the statistics of other authors. This naturally relates chiefly to Elliot's sclero-corneal trephining. Lagrange's cases showed no instance of infection immediate or late; Cridland has reported 70 cases of sclerecto-iridectomy with the same immunity. To the credit of trephining is cited a report by Birch-Hirschfeld of 60 cases without an infection, but others have been less fortunate. Careful study of recent writings on the point will convince anyone, says Lagrange, that the longitudinal resection of the sclera is safer than the use of the trephine. Complications after sclerecto-iridectomy in acute glaucoma have been reported, but the operation is not to be blamed for these, for Lagrange has expressly stated that it is not suited for cases of this type.

As regards visual acuity the greater number of statistical reports are unfortunately found to lack scientific accuracy, so that comparison is difficult. Four favourable exceptions to this statement are cited; they are all Continental. Especial attention is drawn to recent excellent statistics by Lietto-Vollaro of Florence, who has followed Lagrange's method (Bollettino d'Oculistica, June-July, 1922). On the whole the conclusion is that the visual results of trephining have been inferior to those of sclerecto-iridectomy. When the tension is normalized the acuity is usually maintained or bettered, but in a few cases nerve atrophy progresses notwithstanding; on the other hand acuity may be preserved though tension remains a little too high or too low; examples are given. Again, when tension is normalized the field usually retains its previous dimensions or enlarges a little in the nasal area, but it sometimes fails further in this region.

As regards tension accurate reports of results are more numerous, but many cover too short an interval. The figures here cited are not easy to mass for comparison. The conclusion drawn is that sclero-corneal trephining, though it frequently normalizes the tension, does so less frequently and less satisfactorily than sclerecto-iridectomy. 
Summarizing the differences, Lagrange emphasizes not only the higher percentage of success gained by his operation, but its freedom from the disasters of infection, irido-cyclitis, sympathetic ophthalmia, etc., and begs the reader to study the individual reports cited in his article. His final pronouncement is that sclerecto-iridectomy is the right operation for chronic glaucoma, not for acute glaucoma.

Priestley Smith.

\section{II. -A THEORY OF VISION}

\section{Schanz, Fritz (Dresden).-A new theory of vision. Zeitschrift f. Physik., Vol. XII.}

The new theory advocated by Schanz deserves more attention than it seems to have received in British Ophthalmology, and a short note on it may be interesting to the readers of the British Journal of Ophthalmology. As neither the Young-Helmholtz theory of three primary colours with distinct nerve-fibre connections in the retina, nor Hering's of three visual substances with their dissimilation and assimilation has succeeded in establishing itself, we may profitably turn to consideration of a third theory which is based upon purely physical phenomena observed when light acts upon matter everywhere.

Light acts upon matter when it is absorbed by the matter, and the illuminated matter becomes electrified, as is shown at once by the common gold leaf electroscope.

It is only the light of the shorter wave lengths that produces this effect. The waves must actually penetrate into the interior of the molecules, and expel electrons therefrom, which effect the electroscope, and can be collected by wire screens in laboratories. In many cases it is difficult to demonstrate these occurrences, as other chemical changes-often accompanied by oxidation-confuse or obscure the observations. All light rays do not produce the expulsion of electrons, but only the rays of shorter wave lengths, and the velocity of the electrons depends upon the wave length of the light employed. The shorter the wave length the greater the velocity.

Schanz has found that lens albumen, egg albumen, and serum albumen are all chemically altered by the light of shorter wave lengths, and incidentally attributes senile changes in the lens(including cataract) to the action of the ultra-violet rays which never reach the retina in nature as they are absorbed by the lens, and produce in it the phenomena of fluorescence. If the above statements as to the elementary action of light on matter are correct, similar occurrences must accompany every act of vision. Light can only act when it 
is absorbed. It cannot be absorbed by the rods and cones, but it can be, and undoubtedly is, absorbed by the pigment in the retinal epithelium, whence electrons are hurled out and dashed against the rods and cones, which act as the wire-screen used in physical laboratories to collect the electrons, and transmit the electric current through the nerves up to the cerebral centres.

This current is identical with the electric current observed when testing the photo-electric current collected by the wire-screen. Brossa and Kohlrausch have demonstrated that the action current in the optic nerve induced by monochromatic light is characteristic for the different wave lengths of the light employed, and the initial energy is greater the shorter the wave lengths.

Schanz dealt with one objection to the new theory, viz. : "How can albinos see?" in a communication to the joint meeting of the Viennese and German Ophthalmological Societies in August, 1921. In it he stated that after an exhaustive search in the literature he had found no single case reported of an albino's eye without pigment in the retinal epithelium. Schanz brings forward the symptoms in detachment of the retina in support of this theory, but the most brilliant and impressive argument in its favour is the explanation it gives of a difficulty that no other theory has been able to solve, viz., the fact that no eye can detect the difference between a pure spectral colour and one made by a mixture of two or more spectral colours. In this respect our eyes are infinitely inferior to our ears, which can detect each different tone in a chord made up of many different tones.

A little consideration will show how the new theory solves this difficulty. The velocity of the dispelled electrons depends upon the wave lengths of the light-the shorter the wave lengths the greater the velocity, and it can well happen that when light of two different regions of the spectrum is employed, the respective velocities of the electrons may be such that ther become equalized in a brief time-the more rapid pushing on the slower, and the latter reducing the speed of the former, with the result that the average velocity with which they reach the nerve centres is that of electrons due to spectral rays about half-way between the two spectral regions selected.

If, for example, rays are taken from the two regions $\lambda 400 \mu \mu$ and $\lambda 500 \quad \mu \mu$ the average velocity acquired may be that of electrons expelled from rays of $\lambda 450 \mu \mu$, and the colour sensation must then be precisely what is produced by the rays from that spectral region. This is a simple physical explanation for a difficulty which has never yet been solved.

We see white when electrons of all possible velocities act simultaneously on our visual nerves. We also see white if only two groups of electrons are acting if their respective velocities are such 
that that they cannot be equalized as they could be in the above example. When this equalization is impossible we see white and no colours as we do when fusing complementary colours. In the spectrum there are no complementary colours for the rays between $\lambda 500 \mu \mu$ and $\lambda 560 \mu \mu$.

The explanation of this is that electrons of the requisite velocity can only be obtained from ultra-violet rays, and as such rays never reach the retina, being absorbed by the crystalline lens, where they produce the phenomena of fluorescence, they can only be studied in aphakic eyes. This Schanz has done in eyes after cataract extraction, and has found complementary colours in the ultra-violet region for the colours between $\lambda 500 \mu \mu$ and $\lambda 560 \mu \mu$.

J. B. STORY.

\section{III.-OPTIC NERVE AND ACCESSORY SINUSES}

van der Hoeve, J. (Leyden).-Optic nerve and accessory sinuses. Arch. of Ophth., Vol. LI, No. 3, 1922.

van der Hoeve considers the relationship of the optic nerve to the accessory sinuses under four headings: the diagnosis of the optic nerve disease, of the sinus disease, the relation between these two, and, lastly, the treatment.

The optic nerve condition may present itself as choked disc, papillitis, atrophy, or, most frequently, retrobulbar neuritis. The last presents the most difficulty in diagnosis and in this respect the greatest assistance is derived from the examination of the field of vision. Characteristic signs are central scotoma and enlargement of the blind spot, which begin as relative defects, become larger and more intense and blend together, producing a large oval scotoma including and surrounding both fixation point and blind spot. Since these symptoms can be caused by retrobulbar neuritis due to other causes, such as multiple sclerosis, tuberculosis, syphilis, dental infection or rheumatism, the first conclusion is that "The ophthalmologist has in the eye no sign at all to distinguish the origin of a retrobulbar neuritis."

In connection with the second point, the diagnosis of the sinus disease, stress is laid on the value of Rhese's method of radiography of the accessory sinuses which also demonstrates the optic foramen. Even by this method sinus disease may escape detection and the second conclusion is that "The rhinologist cannot say with absolute certainty that a person has no sinus affection," that is to say, without an exploratory operation.

Under the third heading the affection of the nerve is stated to be due to: (1) Direct spread of inflammation. (2) Pressure by the 
walls of a dilated sinus. (3) Damage by toxins, oedema, congestion, etc. The injury done may be reparable or irreparable, the latter being due to atrophic processes following the former.

As regards treatment the necessity for complete examination and free consultation is emphasized. The author is not very definite, but evidently inclines towards the exploration of apparently normal sinuses in cases in which no clear indications are present. Finally, he concludes with a request for statistics to solve the question "Should we operate on the nasal sinuses in optic nerve disease of unknown origin?"

H. M. TRaQuair.

\section{IV.-RELATIONS BETWEEN EYE AND EAR}

van der Hoeve, J. (Leyden).--Relations between eye and ear (including the vestibular organ). Arch. of Ophth., Vol. LI, No. 4, 1922.

The subject is divided into four parts: (1) conditions affecting both eye and ear; (2) eye diseases with ear symptoms; (3) ear diseases with eye symptoms, and (4) the relations between the vestibular organ and the eye.

In the first group blue sclerotics, brittle bones, and otosclerosis, albinism and deafness, Recklinghausen's disease in connection with both second and eighth nerves, and tuberose sclerosis are referred to. Apparently the only eye disease which has been known to cause deafness is sympathetic ophthalmia, and it is suggested that the pigment in the labyrinth is sensitized by the affected eye in the same way as the sympathising eye. Ocular conditions dependent on ear disease include thrombosis of the cavernous sinus and paralysis of the sixth nerve, both due to otitis media. This paralysis is attributed to the local action of toxins, as in the case of retrobulbar neuritis from accessory sinus disease.

The most important ocular-auditory relationship is the ocularvestibular. The author refers to the great scientific as well as clinical interest of this connection to which the bulk of the paper is devoted. Experiments on rabbits are discussed, dealing with the compensatory rotation of the eyes produced by the utriculus otoliths when the head is placed in different positions. In rabbits the compensatory rotation fully corrects the influence of head movement, but in man, although the least rotation of the head produces compensatory rotation of the eyes, the latter is always less than the former. In clinical practice the head must be placed straight in the ophthalmometer in order to get the correct axis of corneal astigmatism, and badly corrected astigmats may assist themselves by holding the head on one side. In man, however, the 
author regards the compensatory corneal rotation as an atavism. Vestibular nystagmus is shortly referred to, and the author concludes with a reference to the difficulty of the subject, which he did not expect all of his audience, with whom the writer of this abstract sympathizes, to understand.

H. M. TRaQuair.

\section{V.-INJURIES OF THE EYE FROM LIGHT}

van der Hoeve, J. (Leyden).--Injuries of the eye from light. (Schädigungen des Auges durch Licht). Klin. Monatsbl. $f$. Augenheilk., 1922, Bd. 68, S.492.

This paper consists of some comments on the views recently expressed by Prof. R. Greeff (Zentralbl. f.d. Ges. Ophth., Bd. VI., Heft. ii.), in connection with light injuries of the eyes. van der Hoeve considers that Greeff has underestimated the effects of light and points out that the average age of cataract patients is some ten years lower in India than in Europe, a feature which he attributes to the action of the ultra-violet rays. The injury to the lens is not direct but due to nutritional disturbance following damage to the ciliary body by rays dispersed by the lens. The relationship of macular degeneration to light and to the presence or absence of the lens is discussed and the author concludes that though it may not be the only cause, ultra-violet light can cause macular disease, especially if the lens is absent.

H. M.'TRAQUAIR.

\section{VI.-OCULAR SPOROTRICHOSIS}

(I) Sobhy Bey, M. - A case of palpebral sporotrichosis (lymphangetic form). Bull. Ophth: Soc. of Egypt, p. 75, 1922.

(1) A man of 30 years consulted M. Sobhy Bey on account of a shallow ulcer in the skin over the lower canaliculus of one eye $3 \mathrm{~mm}$. by $8 \mathrm{~mm}$. in area with a brawny swelling around. In the skin of the lower lid lay a nodule as large as a pea, and there was a second nodule in the upper lid. The conditions had been present for six weeks. The patient also had a sore upon the genitals, and the Wassermann reaction was positive. The glands were enlarged practically all over the body. Smears were taken from ulcers on the lids, and cultures were made on serum agar, blood agar, and simple agar. In one of the smears, stained with 
methylene blue, a long hranching mycelium with two conidia attached to it was found. A young mycelium was grown in a blood agar tube. A smear did show a branching mycelium of voluminous size, and the threads which composed it were ribbonlike, but their branching was at an obtuse angle, and in general they did not look at all like the threads of sporothrix. All attempts to make sub-cultures were negative. Injections of a thin emulsion of the fungus were made under the skin of the ear of a young rabbit, but no lesions resulted. Under treatment by massive doses of potassium iodide the lesions of the lids healed in about six weeks.

S. S.

(2) Gifford, S. R. (Omaha).-Ocular Sporotrichosis. Arch. of Ophthal., November, 1922.

(2) Rare cases of sporotrichosis involving the eye or its appendages have been reported in the main from France or America. In the latter country sporotrichosis is peculiarly a disease of the Mississippi basin. So far as the writer knows, not a single instance is to be found in the British literature. Practically all cases examined bacteriologically have been found to be associated with the Sporothrix Schenkii. The apparent infrequency of the disease is all the more remarkable since the causative organism is stated to have been obtained from several common plants (Toulant). It may affect all parts of the eye under quite different appearances. The affection may be primary or secondary to generalized sporotrichosis. The lesions cause serious destruction of tissue, and may even lead to death. The disease usually takes the form of ulcers of the eyelids or conjunctival sporotrichosis. Diagnosis can be made only by positive culture. Intraocular forms are usually diagnosed by finding lesions elsewhere than in the eye which will yield clinical or cultural evidence. Massive doses of potassium iodide are specific as regards treatment of the disease.

Gifford's first case was in a lad of 8 years, the son of a farmer. The condition had been present for three months before it was diagnosed. A fistula was present just below the left lacrymal sac from which a good deal of pus could be squeezed out. Exploration under a general anaesthetic showed that the opening was $2 / 5$ inch in diameter and extended back $1 \frac{1}{2}$ inches into the left anterior ethmoid cells, which were full of pus. The lacrymal sac was continuous with this opening and over the inner wall the bone was left bare. After removal of the left middle turbinate bone the ethmoid cells were exenterated intranasally, and the passage was enlarged so that a tube could be placed through it into the nose, thus making a modified West operation. The patient was placed under potassium iodide and two months later there was less swelling 
of the parts but still some discharge. The iodide was then stopped on account of gastro-intestinal disturbances. Three months later the opening was present in the skin, but only a few drops of pus could be squeezed out of it. The fistula was cauterized with trichloracetic acid, and tincture of iodine was applied to the deepest parts several times. In spite of this and general treatment with potassium iodide, the sac has continued to fill up with pus and a fairly deep opening in the bone is still present, although the opening into the nose has closed up. After the connection between the conjunctival sac and fistula had been destroyed by cauterization so that the tears could no longer get into the bottom of the fistulous opening, it began to heal better and at the moment of writing seems to be healing from the bottom, although slowly. Smears of the pus at the first examination showed no organisms. Cultures made on plain agar at room temperature after five days contained six discreet white colonies with fine lines radiating from them. Smears from the colonies showed the separate threads with lateral and terminal spores, characteristic of Sporothrix Schenkii. A white rat inoculated with the pure culture died in seven days and the organism was recovered from the peritoneal cavity. A rabbit inoculated in the anterior chamber developed nodules in the iris and cornea with a severe reaction, which subsided after three or four weeks.

The other two cases mentioned by Gifford were probably of sporotrichotic origin although cultures were negative. In one case there was an ulcer involving a portion of the upper lid and in the other case a nodule of the conjunctiva at the outer limbus. It is significant that the lesions healed under treatment by potassium iodide.

\section{BOOK NOTICES}

Diseases of the Eye. By Sir John Herbert Parsons, C.B.E., D.Sc., F.R.C.S., F.R.S. J. \& A. Churchill, 1923. Fourth edition. 21 plates and 326 figures, $660 \mathrm{pp}$. Price 19s. net.

We welcome a new edition of this excellent work which is deservedly popular. The text has been fully revised, and although previously richly illustrated, further coloured plates and diagrams have been added.

This textbook has many outstanding qualities; chief amongst these is the correlation of ophthalmology with allied sciences, notably those of physiology and neurology, which the author's wide scientific knowledge has enabled him to carry out with great success. 